\title{
Detecting Mechanical Abnormalities in Prostate Tissue Using FE-Based Image Registration
}

\author{
Patrick Courtis ${ }^{1}$ and Abbas Samani ${ }^{1,2}$ \\ ${ }^{1}$ Department of Electrical and Computer Engineering, \\ ${ }^{2}$ Department of Medical Biophysics, \\ University of Western Ontario \\ \{pcourtis, asamani\}@uwo.ca
}

\begin{abstract}
An image registration-based elastography algorithm is presented for assessing the stiffness of tissue regions inside the prostate for the purpose of detecting tumors. A 3D finite-element model of the prostate is built from ultrasound images and used to simulate the deformation of the prostate induced by a TRUS probe. To reconstruct the stiffness of tissues, their Young's moduli are varied using Powell's method so that the mutual information between a simulated and deformed image volume is maximized. The algorithm was validated using a gelatin prostate phantom embedded with a cylindrical inclusion that simulated a tumor. Results from the phantom study showed that the technique could detect the increased stiffness of the simulated tumor with a reasonable accuracy.
\end{abstract}

\section{Introduction}

Early detection plays a key role in the prognosis of prostate cancer. A common clinical screening procedure for detecting prostate cancer is the Digital Rectal Exam (DRE). The purpose of the DRE is to detect changes in the stiffness of prostate tissue that may indicate the presence of tumors. Tumors originating in the posterior region of the prostate account for $70 \%$ of all diagnosed cases of prostate cancer and can be detected with the DRE depending on their size and location. The DRE is a qualitative method with low sensitivity, and tumors which are not directly adjacent to the prostatic capsule may not be palpable and must be detected using more sensitive means such as medical imaging analysis. Image guided detection of tumors relies on the assumption that tissue pathology associated with cancer will correspond to changes in the appearance of medical image data. In the case of ultrasound (US) imaging, tumors can be detected based on the relative acoustic properties of normal and malignant tissue: tumors will appear as isolated hyper- (brighter) or hypoechoic (darker) regions in the US image.

Although US can be a valuable tool for locating tumors, its use in the detection of prostate cancer is not well defined. The problem facing US-based detection is the presence of image artifacts or non-malignant diseases like benign prostatic hyperplasia $(\mathrm{BPH})$ that resemble cancerous tumors. In the past, transrectal ultrasound (TRUS) guided biopsy protocols that directly targeted hypo- and hyperechoic regions of prostate tissue were introduced but for the aforementioned reasons, most of these 
biopsies were histologically benign (cancer-free) [1]. As a result, systematic prostate biopsy protocols are now implemented which sample the entire prostate gland. The number of samples in a systematic prostate biopsy can exceed twenty core samples [1]. Currently, the primary role of TRUS in the detection of prostate cancer is to guide biopsy needles into the prostate during the systematic biopsy: specifically, TRUS is used to effectively guide biopsy needles into the posterior region of the prostate, where $80 \%$ of prostate tumors originate [2].

Elastography is an imaging technique that quantifies changes in the mechanical properties of tissue associated with diseases such as prostate cancer [3]. This technique has proven to be very useful in detecting and locating tumors. Although elastography algorithms usually require pre-computed tissue displacement data [4], Miga et al demonstrated that elasography can be performed using FE-based image registration [5] where by tissue displacements and Young's modulus are reconstructed simultaneously. In this paper, we describe an image registration based elastography technique for recovering the Young's modulus of a visually suspicious region in a 3D TRUS volume of the prostate. Similar to elastography techniques of Samani et al [6] and Boctor et al [7] it is assumed that the Young's modulus of each tissue type is constant throughout its volume. This assumption provides anatomical constraints which impose a discrete Young's modulus distribution in the reconstruction. Through the use of this constraint, it is possible to employ a simple optimization procedure (i.e. Powell's method) to perform the Young's modulus reconstruction. This contrasts with previous unconstrained iterative methods, which employ nonlinear least squares algorithms which are generally ill-posed [4;5]. The technique was validated using a gelatin based prostate tissue mimicking phantom with a cylindrical inclusion. The inclusion is meant to simulate a non-palpable prostate tumor.

\section{Methodology}

The technique requires two 3D TRUS volumes: a baseline volume, and a second volume in which the US probe is displaced vertically to deform the prostate. A 3D 20noded brick FE model of the prostate, tumor, rectum, TRUS probe, and surrounding tissue is then used to simulate the deformation induced by the probe. An algorithm for generating the FE model using iso-parametric 20-noded brick elements has been developed. This type of element tends to result in better performance and more accurate FE displacement solutions compared to FE models with a similar number of tetrahedral elements. This is highly advantageous for elastography applications since the displacements computed from the FE-model are being used to reconstruct the mechanical properties of the soft-tissue. Nodal displacements from the FE model are used to register the post-deformed and baseline image volumes. The mechanical properties of each tissue type in the FE model are then varied using Powell's optimization method [8] so that the mutual information (MI) between the transverse and sagittal slices of the registered image volumes is maximized.

\subsection{FE Model Generation}

The mesh generating procedure is based on the concept of transforming rectilinear grids into the shape of the prostate, tumor, and surrounding tissues. The procedure 
involves four major steps. The first three steps, which construct the prostate and tumor are illustrated in Fig. 1. The elements in the surrounding tissue region between the prostate and rectum are generated in the final fourth step.
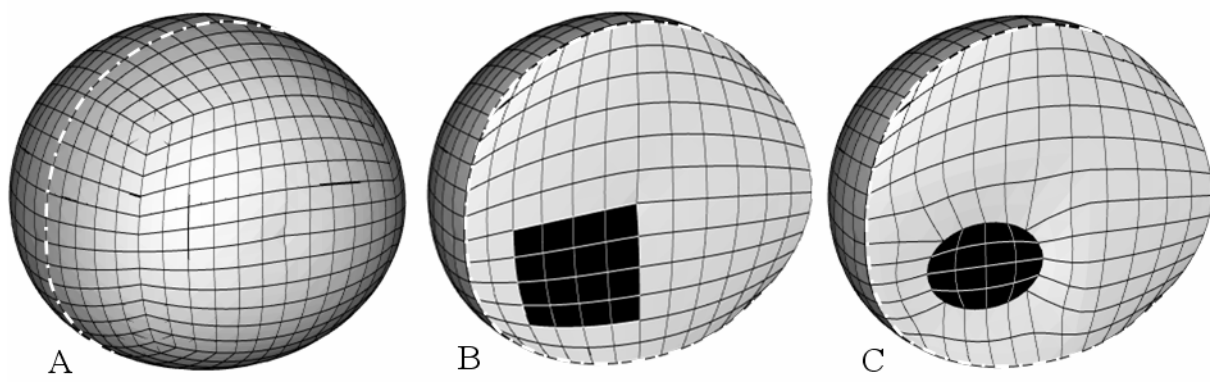

Fig. 1. An illustration of the prostate mesh generating procedure. An ellipsoid is mapped onto the surface of the prostate using a TPS transformation (A). The internal vertices of the mesh are generated using TFI (B). A subsection of the mesh (black) is warped into the shape of the tumor using a CSRBF transformation.

The first step, adapted from the prostate boundary segmentation algorithm developed by $\mathrm{Hu}$ et al [9], uses six control points on the surface of the prostate. Four points are taken from a central transverse 2D slice (two points at the lateral extremes and two points at the posterior and anterior extremes). The other two points are taken from a central sagittal 2D slice at the base and apex of the prostate. The control points are used to generate an ellipsoid [10] that approximates the shape of the prostate. Nodes on the ellipsoid are generated by mapping the six sides of an $N^{3}$ logical grid onto its surface. A thin-plate spline [11] (TPS) transformation warps these nodes so that the six ends of the semi-major axes of the ellipsoid coincide with the corresponding control points.

The nodes inside the prostate are generated by mapping the nodes at the internal vertices of the rectilinear grid to a set of nodes distributed inside the surface of the warped ellipsoid (Fig. 1 B). Using trans-finite interpolation, the position of the nodes on the six surfaces of the prostate are used to compute the position of the nodes inside the prostate according to the 3D vector-valued bilinear blended mapping defined in Knupp et al [12]. One advantage of this approach is that element connections determination is straight forward as it is done using the nodes of the rectilinear grid.

In step three, a tumor is formed inside the prostate mesh. In this research, tumors are modeled as cylinders embedded inside the prostate tissue. This is done by replacing a subzone (black area) with a cylindrical shaped grid as illustrated in Fig. 1 - C. To avoid excessive distortion of the mesh resulting from warping the subzone to the tumor shape, the nodes surrounding the tumor are then repositioned using compact support radial basis functions (CSRBF). With CSRBFs one can specify a distance $r$ beyond which all the transformation parameters are zero. Here, $r$ is taken as the shortest distance between the surface of the tumor and the surface of the prostate. This enables a smooth transformation of a group of elements into the shape of the tumor while maintaining the original boundary of the prostate mesh. 
After the elements that form the prostate and tumor have been generated, the elements that form the tissue surrounding the prostate and rectum are generated. First, a rectilinear grid $\mathbf{x}(i, j, k)$ is defined where $i, j=0,1, \ldots, N+2, k=0,1, \ldots, 2 N+2$, and $N$ is the dimensionality of the grid that was used to form the vertices of the prostate. The nodes on the surface of $\mathbf{x}$ are mapped onto the boundary of the external tissue region. The internal vertices $\mathbf{x}=(1 \leq \mathrm{I} \leq N+1,1 \leq \mathrm{j} \leq N+1, N-1 \leq \mathrm{k} \leq 2 N+1)$ are vertices of the prostate mesh that were generated in steps one through three. The surface of the vertices $\mathbf{x}=(1 \leq \mathrm{I} \leq N+1,1 \leq \mathrm{j} \leq N+1,1 \leq \mathrm{k} \leq N+1)$ is mapped onto the surface of the rectum takes the shape of a cylinder. After the rectum is defined, a set of additional vertices are generated in between the prostate, rectum, and surrounding tissue. The positions of these new vertices are determined using a tri-linear interpolation calculation based on the position of eight nodes from the original grid surrounding each vertex.

\subsection{Prostate Phantom Validation}

A prostate tissue mimicking phantom was constructed from a mixture of gelatin, water, n-propanol and formaldehyde [13]. The mechanical and acoustic scattering properties of the phantom were varied by altering the respective concentrations of gelatin. The relative mechanical properties of the prostate, tumor, and surrounding region within the phantom were adjusted to reflect the literature values [3]. To enhance image contrast, varying amounts of cellulose agent were added to the different parts of the phantom: $0.02 \mathrm{~g} / \mathrm{mL}$ in the surrounding material and $0.01 \mathrm{~g} / \mathrm{mL}$ in the prostate. No cellulose was added to the inclusion. The Young's modulus of the gelatin based prostate, inclusion, and surrounding material were measured independently from uniaxial load test data on cylindrical gelatin samples [14]. The phantom was manufactured in a Plexiglas container where the front, left and right sides could be removed to facilitate axial deformation induced by the TRUS probe. A thin layer of acoustic damping rubber was placed on the Plexiglas above the prostate in order to identify the top of the container in the US image volumes. The phantom was manufactured with a cylindrical hole below the prostate, approximately the diameter of the TRUS probe, to simulate the rectum and allow for insertion of the probe. A diagram of the phantom assembly is shown in Fig. 2. B-mode US image volumes of the phantom assembly were acquired using a 3D TRUS system developed by Fenster et al [15]. The reconstructed image volumes have a pixel spacing of 0.21 $\mathrm{mm}$ in the coronal and axial plane and $0.20 \mathrm{~mm}$ in the sagittal plane. Deformed phantom images were acquired by rescanning the phantom after an upward displacement was applied to the TRUS probe. A schematic of the phantom and a photograph of the 3D TRUS system are shown in Fig 2.

The baseline TRUS volume was acquired with the probe resting flush against the cylindrical rectum of the phantom, and a second volume was acquired after a vertical displacement was applied to the TRUS probe. The displacement of the TRUS probe was determined visually by manually aligning the echo signals on the upper surface of the phantom container in the axial image plane. A frictionless contact FE formulation that modeled the deformation induced by the vertical displacement of the TRUS probe was implemented with ABAQUS 6.6 [16] and used to perform an initial FE analysis in order to estimate the displacement boundary conditions that were induced by the probe along the surface of the rectum. This initial FE analysis assumes that the phantom mesh is composed of a single Young's modulus. Subsequent FE 
calculations during the reconstruction algorithm used the displacements computed from this contact analysis as boundary conditions, eliminating the need to perform contact analysis throughout the optimization process.

Using Powell's methods, the mechanical properties of the prostate and inclusion were varied and new displacement from the FE-model were computed, so that the MI between the two orthogonal slices in the registered image volumes was maximized. The Young's modulus of the surrounding tissue is assigned its experimental value. The MI was computed using two windowed and $3 \times 3$ median filtered regions of interest (ROIs) surrounding the prostate on axial and sagittal slices through the centre of the phantom. Median filtering the US image is used to reduce the number of local maxima in the parameter search space. The two lateral extremes of the prostate were not included in the axial ROI due to the concern that poor lateral resolution at these points in the image could have an adverse affect on the MI calculation.
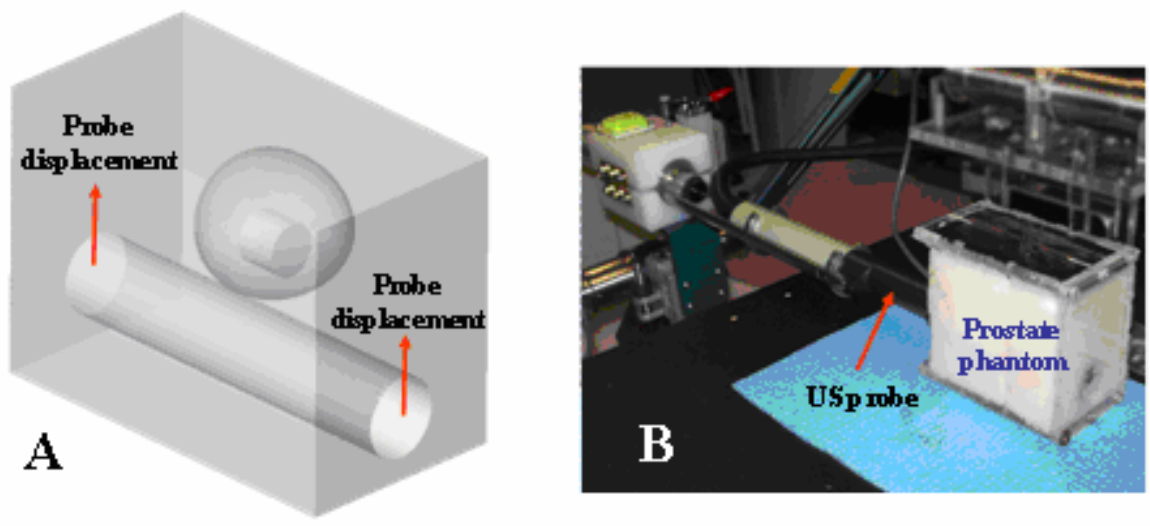

Fig. 2. A schematic diagram of the prostate phantom assembly (A) and a photograph of the TRUS apparatus (B)

\section{Results and Discussion}

Fig. 3 shows the results of FE analysis within the axial and sagittal planes. The simulated post-deformed images that maximized the mutual information metric are shown in Fig. 4. The subtracted 8-bit images (Fig. 4 A3, B3) were windowed and leveled so that the minimum and maximum pixel value corresponds to 0 and 255 respectively. The reconstructed Young's modulus values are presented in Table 1. The subtracted images in Fig. 4 demonstrate that the optimal Young's modulus values result in well registered image volumes. By inspection of Table 1, the reconstruction determined that the cylindrical tumor is stiffer than the surrounding phantom tissue and that the reconstructed values are reasonably close to the Young's modulus values calculated from the uniaxial load test data. The reconstructed values of $\mathrm{E}_{2}, \mathrm{E}_{3}$ differ by $23 \%$ and $19 \%$ from their respective independently measured values. The parameter of most interest: the relative stiffness of the simulated tumor to the surrounding prostate mimicking tissue $\left(E_{3} / E_{2}\right)$, was reconstructed with reasonable accuracy, differing by $5.6 \%$ from its experimentally determined value. 


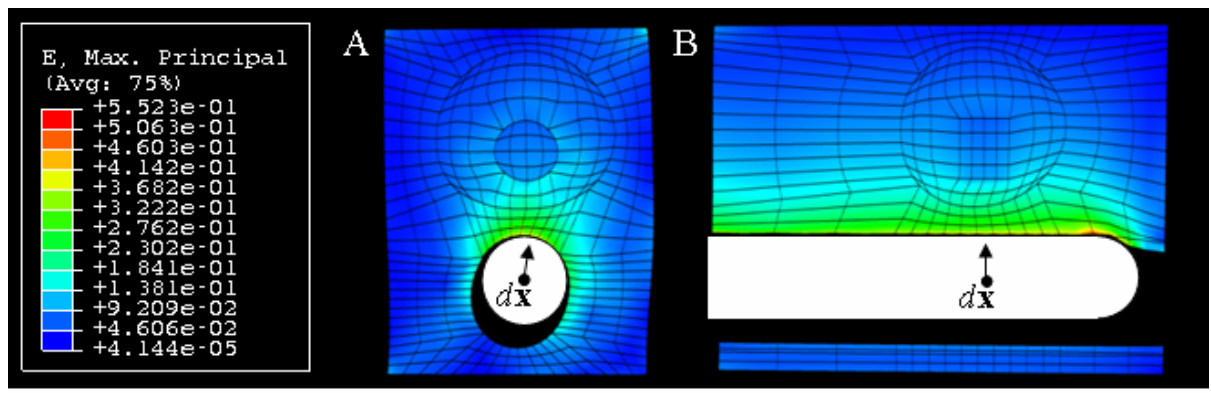

Fig. 3. Tissue maximum principal strain induced by the TRUS probe in the axial (A) and sagittal (B) planes obtained from FE analysis

Table 1. A comparison of the Young's modulus values reconstructed by the image registration algorithm. $E_{1}, E_{2}, E_{3}$, are the Young's modulus values of the surrounding, prostate, and cancer (tumor) mimicking tissue, respectively.

\begin{tabular}{ccccccc}
\hline & $\mathbf{E}_{\mathbf{1}}[\mathbf{k P a}]$ & $\mathbf{E}_{\mathbf{2}}[\mathbf{k P a}]$ & $\mathbf{E}_{\mathbf{3}}[\mathbf{k P a}]$ & $\mathbf{E}_{\mathbf{3}} / \mathbf{E}_{\mathbf{1}}$ & $\mathbf{E}_{\mathbf{3}} / \mathbf{E}_{\mathbf{2}}$ & $\mathbf{E}_{\mathbf{2}} / \mathbf{E}_{\mathbf{1}}$ \\
\hline Experimental & 8.5 & 12.9 & 22.6 & 2.7 & 1.8 & 1.5 \\
Reconstructed & 8.5 & 9.9 & 18.4 & 2.2 & 1.9 & 1.2 \\
\hline
\end{tabular}

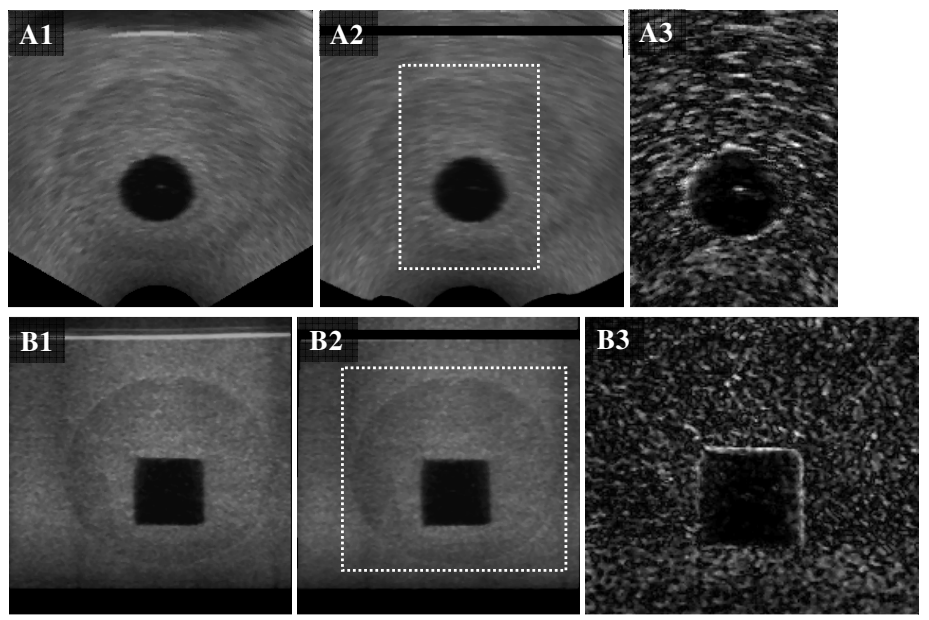

Fig. 4. A1 and B1 are simulated and A2, B2 are target- axial and sagittal post-deformed images respectively. Images A3 and B3 are the windowed and leveled subtracted images of each pair within the windowed area indicated by the white box in B1 and B2. 
One major source of error is the measurement of the boundary condition induced by the TRUS probe. In this research it was assumed that only translational displacements in the axial plane were applied to the probe, however, observing the tumor in the subtracted image in Fig. 4 B2, it appears that there is some bending of the apparatus used to hold the TRUS probe while it is pressed against the cylindrical rectum of the phantom. Bending of the apparatus could be observed while the phantom was being deformed and the probe had to be leveled manually using a leveling tool before each US volume was acquired. An additional source of error is the FE calculation. It is possible that the relative number of elements in each of the three material domains had an effect on the reconstructed values. Segmentation error along the surface of the prostate is a third and additional source of error since the warped ellipsoid used to approximate the boundary of the prostate may not pass exactly over the surface of the prostate.

\section{Conclusions and Future Work}

An image registration based-elastography algorithm for evaluating the mechanical properties of a visually suspicious region in the prostate was designed and validated using a gelatin based tissue phantom. Unlike traditional elastography that relies on displacement data as input, this technique used the MIE paradigm to formulate elastography as an image registration problem.

The relative value of the reconstructed Young's modulus of the tumor mimicking inclusion with respect to the prostate phantom tissue $\left(E_{3} / E_{2}\right)$ was reconstructed with an error of $\sim 6 \%$. The results are encouraging and show good potential for using this technique in prostate cancer detection. The error in the absolute values of $E_{2}$ and $E_{3}$ with respect to the surrounding tissue $(\sim 23 \%)$ is likely caused by mis-registration of the US volumes caused by rotational motion of the apparatus that held the TRUS probe.

Future work will involve either designing an apparatus that can accurately measurethe transformation of the TRUS probe when it is used to deform the prostate, or, incorporating the transformation parameters of probe as additional variables in the Powell's optimization procedure. Measuring the probe transformation parameters directly is preferable since adding additional parameters to the optimization will only increase the time required to perform the Young's modulus reconstruction. Extending the meshing algorithm to include accurate segmentation of real prostate boundaries and tumors will also be pursued.

\section{References}

[1] Raja, J., Ramachandran, N., Munneke, G., Patel, U.: Current status of transrectal ultrasound-guided prostate biopsy in the diagnosis of prostate cancer. Clin. Radiol. 61(2), $142-153$ (2006)

[2] McNeal, J.E., Redwine, E.A., Freiha, F.S., Stamey, T.A.: Zonal distribution of prostatic adenocarcinoma. Correlation with histologic pattern and direction of spread. Am. J. Surg. Pathol. 12(12), 897-906 (1988) 
[3] Krouskop, T.A., Wheeler, T.M., Kallel, F., Garra, B.S., Hall, T.: Elastic moduli of breast and prostate tissues under compression. Ultrason. Imaging 20(4), 260-274 (1998)

[4] Kallel, F., Bertrand, M.: Tissue elasticity reconstruction using linear perturbation method. Medical Imaging, IEEE Transactions on 15(3), 299-313 (1996)

[5] Washington, C.W., Miga, M.I.: Modality independent elastography (MIE): a new approach to elasticity imaging. IEEE Trans. Med Imaging 23(9), 1117-1129 (2004)

[6] Samani, A., Bishop, J., Plewes, D.B.: A constrained modulus reconstruction technique for breast cancer assessment. IEEE Trans. Med Imaging 20(9), 877-885 (2001)

[7] Boctor, E., de Oliveira, M., Choti, M., Ghanem, R., Taylor, R., Hager, G., Fichtinger, G.: Ultrasound monitoring of tissue ablation via deformation model and shape priors. Med Image Comput. Comput. Assist. Interv. Int. Conf. Med Image Comput. Comput. Assist. Interv. 9(2), 405-412 (2006)

[8] Press, W.H., Teukolsky, S.A., Vetterling, W.T., Flannery, B.P.: Numerical Recipes in C, 2nd edn., pp. 36-41. Press Syndicate of the University of Cambridge, Cambridge, London, England (2002)

[9] Hu, N., Downey, D., Fenster, A., Ladak, H.M.: Prostate boundary segmentation from 3D ultrasound images. Med. Phys. 30(7), 1648-1659 (2003)

[10] Tietze, H.: Famous Problems of Mathematics: Solved and Unsolved Mathematics Problems from Antiquity to Modern Times, p. 28. pp. 40-41. Graylock Press, New York (1965)

[11] Bookstein, F.L.: Principal Warps: Thin Plate Spline and the Decomposition of Deformations. IEEE Transactions on Pattern Analysis 11(6), 567-585 (1989)

[12] Knupp, P.M., Steinberg, S.: Fundamentals of Grid Generation. CRC Press, Boca Raton (1994)

[13] Hall, T.J., Bilgen, M., Insana, M.F., Krouskop, T.A.: Phantom materials for elastography. Ultrasonics, Ferroelectrics and Frequency Control, IEEE Transactions on 44(6), 13551365 (1997)

[14] Samani, A., Bishop, J., Luginbuhl, C., Plewes, D.B.: Measuring the elastic modulus of ex vivo small tissue samples. Phys. Med. Biol. 48(14), 2183-2198 (2003)

[15] Tong, S., Downey, D.B., Cardinal, H.N., Fenster, A.: A three-dimensional ultrasound probe imaging system. Ultrasound in Medicine and Biology 22, 735-746 (1996)

[16] Hibbit, K.S.: ABAQUS: Theory Manual. Pawtucket, RI (1998) 